\title{
Information on oral cancer encourages primary-care patients to accept oral cancer screening and reduces associated anxiety
}

\author{
Does information about oral cancer encourage primary-care patients to accept \\ oral cancer screening?
}

\begin{abstract}
Humphris GM, Ireland RS, Field EA. Randomised trial of the psychological effect of information about oral cancer in primary care settings. Oral Oncol 2001; 37:548-552
\end{abstract}

Design A randomised controlled trial was carried out in medical and dental primary-care settings.

Intervention A total of 800 patients was randomised into leaflet or non-leaflet groups. Patients randomised to the leaflet group were given a leaflet on oral cancer to read. All patients were then asked to complete a questionnaire.

Outcome measure To assess intention to screen, patients were asked how likely they were to agree to a check-up for oral cancer. Patients selected their answer from a seven-point scale ranging from "extremely unlikely" to "extremely likely". Anxiety about having an oral cancer check-up was assessed on a five-point scale ranging from "not anxious" to "extremely anxious". The perceived risk of finding they had mouth cancer in the next year was recorded by patients on a seven-point scale ranging from "extremely unlikely" to "extremely likely". Knowledge of oral cancer was assessed on a 36-item scale.

Results Those in the leaflet group were significantly more likely to agree to an oral cancer screen $(P<0.001)$ and reported significantly less anxiety $(P<0.05)$ than the control group. Those who had achieved higher scores on the knowledge questionnaire (ie, greater knowledge) were more likely to agree to a screen. Intention to screen was not affected by level of perceived risk, practice type, age and gender.

Conclusions Giving primary-care patients information on oral cancer encouraged them to accept an oral cancer screen and reduced the level of anxiety associated with it.

\section{Commentary}

This paper is an important contribution to the ongoing research and discussion of the appropriateness of screening for oral and pharyngeal cancers. The Oral Health Group of the Cochrane Collaboration is currently performing a systematic review of screening programmes for the early detection and prevention of oral cancer (see www.cochrane.org/cochrane/revabstr/g080index.htm) and systematic reviews on the same subject have recently been performed by other groups. ${ }^{1,2}$ The latter two reviews each concluded that there is insufficient evidence to recommend screening in the general population ${ }^{1,2}$ although "opportunistic" screening of high-risk individuals at yearly intervals by both dentists and physicians should be considered. ${ }^{2}$ The limited research in this field suggests that one of the main reasons why screening for oral cancer is apparently ineffective is ignorance of the disease and of the need to screen both on the part of the public ${ }^{3,4}$ and health professionals. ${ }^{4,5}$ If this is the case, we need research to demonstrate the effectiveness of interventions in order to change the public's and health professionals' knowledge and attitudes towards screening for oral cancer, before embarking on studies investigating the effectiveness of screening. In this context, the reviewed paper advances our understanding of this field of healthcare research.

As part of the same research project, Humphris et al. (2000) already demonstrated that a short leaflet concerning oral cancer could improve knowledge of the matter in members of the public. ${ }^{6}$ This second paper from the project aimed to investigate the effect of the same leaflet on people's intention to be screened for oral cancer and their anxiety about screening.

The study used a randomised controlled trial design in which randomisation units were sessions in the primary healthcare settings when study recruitment and data collection occurred. For instance, a Monday morning at study centre A may have been randomised as a control session so all subjects recruited that morning did not receive the information leaflet, whereas a Tuesday afternoon at centre B was randomised as a test session, so that all recruits that afternoon received the leaflet. Given the study setting, this technique has the important advantage of preventing contamination of test and control subjects. It nevertheless raises two questions that were not addressed in the paper. First, exactly how did the randomisation occur? Secondly, what was the wording of the project description for the consent process as randomisation occurred prior to consent, rather than afterwards as most often happens? The paper reported the results of the randomisation, stating that the influence of background variables on the experimental intervention was successfully removed, with the exception of gender, which was subsequently controlled for in the analyses.

Beyond randomisation, another important design issue is blinding. Unfortunately, blinding was not possible for this study because of the nature of the intervention and the fact that the primary outcomes were subject self-evaluations. These primary outcomes (intention to screen and anxiety about oral cancer) were both
Address for correspondence: GM Humphris, Department of Clinical Dental Services, School of Dentistry, Pembroke Place, University of Liverpool, Liverpool L69 3GB, UK. E-mail: cpsy1@liverpool.ac.uk 
measured using single items, each with Likert response scales. The test-retest reliability of these measures, checked in university undergraduate students, was good. This rapid, convenient technique was presumably used because of the data-gathering setting (a busy primary healthcare setting) but means it is difficult to compare, for instance, anxiety ratings from this study with those of studies using other, better-recognised measures.

The results of these evaluations were presented both in terms of mean scores and percentages of each category in the respective Likert scales. This is useful because, although it is difficult to interpret the somewhat abstract mean scores and any differences between the two groups (even if they are statistically different), data presented on the proportion of people who were, for example, "extremely likely" to go for a screening in the test group (47.8\%) and control group $(36.0 \%)$ enable the reader to see the clinical significance of the results as well. The authors found a significantly increased intention to screen and reduced anxiety in the test group compared with the control group. One criticism over the presentation of the results, however, is that the reader is not given the preintervention intention to screen and anxiety scores which, given the one-item nature of each of these outcomes, would have been feasible and would have demonstrated that the pre-intervention scores were the same as the control group.

As a whole the results reported in this paper and its sister report ${ }^{6}$ suggest that a simple leaflet provided to the general public in a primary healthcare setting can improve knowledge about oral cancer, increase the intention to go for screening and reduce the anxiety about screening. Nevertheless, as the authors state themselves, the next and very important stage is to investigate whether such an intervention can alter people's behaviour, so that more individuals present for oral cancer screening. This is obviously a crucial step in the progress of research towards understanding how to provide effective oral cancer screening programmes and hopefully reduce the morbidity and mortality resulting from this disease.

\section{Practice point}

- A simple leaflet provided in a primary healthcare setting can change knowledge in members of the public about oral cancer, increase the intention to go for screening and reduce anxiety about screening.

\section{Paul Allison}

Faculty of Dentistry, McGill University, Montreal, Canada

1. Truman $\mathrm{BI}$, Gooch BF, Sulemana I et al. Reviews of evidence on interventions to prevent dental caries, oral and pharyngeal cancers, and sports-related craniofacial injuries. Am J Prevent Med 2002; 23(Suppl. 1):21-54.

2. Hawkings RJ, Wang EEL, Leake IL. Preventive health care, 1999 update: prevention of oral cancer mortality. J Can Dent Assoc 1999; 65:617 [abridged version; for full version see www.cda-adc.ca/jcda/vol-65/issue-11/617.htm].

3. Warnakulasuriya $K$, Harris $C$, Scarrott $D$, et al. An alarming lack of public awareness towards oral cancer. Br Dent J 1999; 187:319-322.

4. Horowitz AM, Nourjah PA. Factors associated with having oral cancer examinations among US adults 40 years of age and older. J Publ Health Dent 1996; 56:331-335.

5. Goodman HS, Yellowitz JA, Horowitz AM. Assessing physicians' and dentists' oral cancer knowledge, opinions and practices. J Am Dent Assoc 1995; 126:53-60.

6. Humphris G, Ireland R, Field E. Immediate knowledge increase from an oral cancer information leaflet in primary health service attenders: a randomised controlled trial. Oral Oncol 2001; 37:99-102.

Evidence-Based Dentistry (2003) 4, 68-69.

doi:10.1038/sj.ebd.6400200

\section{UK Mouth Cancer Awareness Week 9-15th November 2003}

